\title{
NON-HOPFIAN GROUPS WITH FULLY INVARIANT KERNELS. I
}

\author{
BY
}

\author{
MICHAEL ANSHEL( $(1)$
}

ABSTRACT. Let $\mathcal{\&}$ consist of the groups $G(l, m)=\left(a, b ; a^{-1} b^{l} a=b^{m}\right)$ where $|l| \neq 1 \neq|m|, l m \neq 0$ and $l, m$ are coprime. We characterize the endomorphisms of these groups, compute the centralizers of special elements and show that the endomorphism $a \rightarrow a, b \rightarrow b^{l}$ is onto with a nontrivial fully invariant kernel. Hence $G(l, m)$ is non-Hopfian in the 'fully invariant sense.'

Our purpose is to prove the results announced in [1], concerning the endomorphisms of the non-Hopfian one-relator groups $\mathcal{Q}$ found in [2] and isolated by G. Baumslag in [3]. $\&$ consists of the groups $G(l, m)$ presented by $(a, b$; $a^{-1} b^{l} a=b^{m}$ ) where $|l| \neq 1 \neq|m|, l m \neq 0$ and $l, m$ are coprime. Let $G^{\prime}$ denote the normal closure of $b$ in $G(l, m)$ and $N$ the kernel of the endomorphism $\eta$ : $a \rightarrow a, b \rightarrow b^{l}$. We will prove

Theorem 1. If $r: a \rightarrow A, b \rightarrow B \neq 1$ defines an endomorphism of $G(l, m)$ then

(1) $B$ is in $G^{\prime}$ and can be written in the form $B=D^{-1} b^{k} D(D$ in $G(l, m)$ ).

(2) $D A D^{-1}=c a$ where $c$ is in the centralizer of $b^{l k}$ in $G^{\prime}$.

Theorem 2. $N$ is a proper fully invariant subgroup of $G(l, m)$ sucb that $G(l, m) / N$ is isomorphic to $G(l, m)$.

A general reference for the proofs of these theorems is [4].

1. Basic lemmas.

Lemma 1. $\eta: a \rightarrow a, b \rightarrow b^{l}$ defines an onto endomorphism of $G(l, m)$ with nontrivial kernel $N$ where $N$ is the normal closure of the subgroup generated by

$$
W(a, b)=\left([b, a]^{t} b^{s}\right) b^{-1}
$$

and

$$
V(a, b)=a^{-1} b a\left([b, a]^{t} b^{s}\right)^{-m}
$$

sucb that $(m-l) t+l s=1$.

Received by the editors July 15, 1971 .

AMS 1970 subject classifications. Primary 20F05; Secondary 20E05.

Key words and phrases. Groups with one defining relation, endomorphisms of groups, fully invariant subgroups, characteristic subgroups, reduced free groups, relatively free groups.

(1) These results may be found in the author's doctoral dissertation written while he was an N.S.F. Cooperative Fellow at Adelphi University.

Copyright $\odot 1972$, American Mathematical Society 
Proof. That $\eta$ is onto and $N \neq 1$ follow from [2]. Let $H$ be presented by $\left(a, b ; a^{-1} b^{l} a=b^{m}, W(a, b)=V(a, b)=1\right)$. Define $\alpha: G \rightarrow H$ where $\alpha: a \rightarrow a, b \rightarrow b$ and $\beta: H \rightarrow G$ where $a \rightarrow a, b \rightarrow b^{l}$. Now observe that $\beta$ is an isomorphism (with inverse $\bar{\beta}: H \rightarrow G, \bar{\beta}: a \rightarrow a, b \rightarrow[b, a]^{t} b^{s}$ ) and that $a \beta=\eta$. Hence ker $\eta=$ $\operatorname{ker} \alpha \beta=N$.

The proofs of Lemmas 2 and 3 are immediate consequences of the ReidemeisterSchreier rewriting process [4].

Lemma 2. Let $G^{\prime}$ be the normal closure of $b$ in $G(l, m) . G^{\prime}=\left(\cdots x_{i} \cdots\right.$; $x_{i}^{l}=x_{i+1}^{m}$, where $i$ runs through the integers) and $x_{i}=a^{i} b a^{-i}$ in $G(l, m)$.

Lemma 3. In $G^{\prime}$ we bave for $k>0$,

$$
\begin{aligned}
& \left(x_{j}\right)^{l^{k}}=\left(x_{j+k}\right)^{m^{k}} \text { for any } j, \\
& \left(x_{i}\right)^{m^{k}}=\left(x_{i-k}\right)^{l^{k}} \text { for any } i .
\end{aligned}
$$

Let $G^{\prime \prime}$ denote the commutator subgroup of $G^{\prime}$ and $Q(l, m)$, the additive subgroup of the rationals, generated by $(l / m)^{i}$ where $i$ runs through the integers. $G / G^{\prime \prime}$ is isomorphic to the obvious split extension of $Q(l, m)$ by an infinite cyclic group. Explicitly

Lemma 4. $G^{\prime} / G^{\prime \prime}$ is isomorphic to $Q(l, m)$ under the map $\gamma: x_{i} \rightarrow(l / m)^{i}$.

2. Conjugacy in $G(l, m)$. Here we examine certain subgroups of $G^{\prime}$ and their length functions to establish certain conjugacy properties.

For $j<k$ let

$$
\begin{aligned}
A(j, k) & =\left(x_{j}, \cdots, x_{k} ; x_{j}^{l}=x_{j+1}^{m}, \cdots, x_{k-1}^{l}=x_{k}^{m}\right), \\
A(j, j) & =\left(x_{j} ;\right) \quad \text { the infinite cyclic group, } \\
A(-\infty, k) & =\left(\ldots x_{k-1}, x_{k} ; x_{i}^{l}=x_{i+1}^{m}, i<k\right), \\
A(j,+\infty) & =\left(x_{j}, x_{j+1}, \ldots, x_{i}^{l}=x_{i+1}^{m}, j \leq i\right) .
\end{aligned}
$$

The left factorization of $A(j, k)$ is given by the free product with amalgamation

$$
A(j, k-1) * A(k, k), \quad x_{-1}^{l}=x_{k}^{m},
$$

and the right factorization of $A(j, k)$ by

$$
A(j, j) * A(j+1, k), \quad x_{j}^{l}=x_{j+1}^{m} .
$$

For $x$ in $A(j, k)$ let $\lambda_{j_{k}}(x)$ denote the representative length w.r.t. the left factorization and $\rho_{j_{k}}(x)$ the representative length w.r.t. the right factorization 
[4]. We will refer to these functions respectively, as the left and right length functions of $A(j, k)$. Elements cyclically reduced w.r.t. these length functions will be referred to as left (resp. right) cyclically reduced. For $p \leq j, A(p, k)$ will be called a left extension of $A(j, k)$ and for $q \geq k, A(j, q)$ will be called a right extension of $A(j, k)$. For $x$ in $G^{\prime}$ and $n \geq 0, a^{-n} x a^{n}$ (resp. $a^{n} x a^{-n}$ ) is called a left (resp. right) translate.

In proving results where a dual statement arises by substituting 'right' for 'left' we omit when obvious the proof of the dual. We will refer to Theorem 4.6 in [4] as Theorem 4.6.

The proofs of Corollaries 1 and 2 are immediate consequences of the properties of representative length functions [4].

Corollary 1. If $x$ is left (resp. right) cyclically reduced in $A(j, k)$ then $x$ is left (resp. right) cyclically reduced in all left (resp. right) extensions of $A(j, k)$ and $\lambda_{j_{k}}(x)=\lambda_{p_{k}}(x)$ (resp. $\rho_{j_{k}}(x)=\rho_{j_{q}}(x)$ ).

Corollary 2. If $x$ is left (resp. right) cyclically reduced in $A(j, k)$ and $\lambda_{j_{k}}(x) \geq 2$ (resp. $\rho_{j_{k}}(x) \geq 2$ ). Then for $|n| \geq 1, x^{n}$ is left (resp. right) cyclically reduced in $A(j, k)$ and $\lambda_{j_{k}}\left(x^{n}\right)=|n| \lambda_{j_{k}}(x)$ (resp. $\rho_{j_{k}}\left(x^{n}\right)=|n| \rho_{j_{k}}(x)$ ).

Corollary 3. If $x$ is left (resp. right) cyclically reduced in $A(j, k)$ and $\lambda_{j_{k}}(x) \geq 2$ (resp. $\rho_{j_{k}}(x) \geq 2$ ) then for all left (resp. right) translates $y$ of $x^{l}$ in a given left (resp. right) extension of $A(j, k), y$ is not conjugate to $x^{m}$ in that extention.

Proof. For $y=x^{l}$, by Corollaries 1 and $2, \lambda_{p_{k}}\left(x^{l}\right) \neq \lambda_{p_{k}}\left(x^{m}\right)$ so, from Theorem 4.6, $y$ and $x^{m}$ are not conjugate in any left extension. For $y=$ $a^{-n} x^{l} a^{n}, \lambda_{p_{k}}(y) \leq 1$ and again by Theorem 4.6 the result follows.

An immediate consequence of Theorem 4.6 is

Lemma 5. Let $G=A *_{H} B, H \subset$ Center $(B)$. If $g$ is cyclically reduced in $G$ and $g$ is conjugate to $b$ in $H$ then $g$ is conjugate in $A$ to $b$.

Corollary 4. If $x$ is left (resp. right) cyclically reduced and $\lambda_{j_{k}}(x) \geq 2$ (resp. $\rho_{j_{k}}(x) \geq 2$ ) then $x$ is not conjugate in any right (left) extension $A(j, q)$ (resp. $A(p, q)$ ) to an element in $A(q, q)$ (resp. $A(p, p))$.

Proof. For $q=k$, we have from Theorem 4.6 and Corollary 2 that $x^{n},|n| \geq 1$, is not conjugate in $A(j, k)$ to an element in $A(k, k)$. Suppose the result for $q \leq k+i$ and let $q=k+i+1$ and note $\lambda_{j_{q}}(x) \leq 1$. If $x$ is conjugate in $A(j, q)$ to an element $y$ in $A(q, q), y$ not conjugate in $A(j, q)$ to an element of the amalgamated subgroup $H$ generated by $x_{q}^{m}$ then, by Theorem 4.6, x,y are in $A(q, q)$ and conjugate there. Thus $x=x_{q}^{\mu}, \mu \neq 0$. Now by Lemma 3, 


$$
x_{q}^{m^{i+1}}=x_{q-(i+1)}^{l^{i+1}}=x_{k}^{l i+1}
$$

so

$$
x^{m^{i+1}}=\left(x_{k}^{l i+1}\right)^{\mu}
$$

which is contrary to $x^{n}$ not in $A(k, k)$ for $|n| \geq 1$. Thus if $x$ is conjugate in $A(j, q)$ to an element $y$ in $A(q, q)$ then $y$ is conjugate in $A(j, q)$ to an element $b$ in $H$. Since $x$ is cyclically reduced in $A(j, q)$ and $x$ is in $A(j, k+i)$, by Lemma $5, x$ would be conjugate to $b$ in $A(j, k+i)$ contrary to our inductive hypothesis.

Corollary 5. If $x$ and $y$ are left (resp. right) cyclically reduced in $A(j, k)$, $\lambda_{j_{k}}(x) \geq 2$ (resp. $\rho_{j_{k}}(x) \geq 2$ ) then $x$ is conjugate to $y$ in a right (resp. ieft) ex. tension of $A(j, k)$ implies $x$ conjugate to $y$ in $A(j, k)$.

Proof. For $q=k$ the result holds. Suppose the result holds for $q \leq k+i$ and let $q=k+i+1$. By Corollary $4, x$ is not conjugate in $A(j, q)$ to an element in $A(q, q)$. Hence, by Theorem $4.6, x$ is conjugate to $y$ in $A(j, k+i)$. Applying the inductive hypothesis yields $x$ conjugate to $y$ in $A(j, k)$.

Corollary 6. If $x$ is not conjugate in $G^{\prime}$ to an element in $A(i, i)$ for any $i$ and $x$ is in $A(j, k)$ then $j<k$ and (1) there exists $a j^{\prime}$ and an element $y$ sucb that $j \leq j^{\prime}<k$, y is cyclically reduced in $A\left(j^{\prime}, k\right), \rho_{j_{k}^{\prime}}(y) \geq 2$ and $x$ is conjugate to $y$ in $A(j, k)$; (2) there exists a $k^{\prime}$ and an element $z$ sucb that $j<k^{\prime} \leq$ $k, z$ is cyclically reduced in $A\left(j, k^{\prime}\right), \lambda_{j_{k}}(z) \geq 2, x$ is conjugate to $z$ in $A(j, k)$.

Proof. Clearly $j<k$. For $k-j=1$ the result holds since $x$ is not conjugate in $G^{\prime}$ to an element of either $A(j, j)$ or $A(j+1, j+1)$. Thus, cyclically reducing $x$ in $A(j, j+1)$ yields $y$ and setting $y=z$ yields our construction. Suppose the result is true for $k-j \leq n$ and let $k-j=n+1$. Now cyclically reduce $x$ w.r.t. the right factorization of $A(j, k)$ to $y^{\prime}$. If $\rho_{j_{k}}\left(y^{\prime}\right) \geq 2$ let $y=y^{\prime}$. Otherwise $y^{\prime}$ is in the factor $A(j+1, k)$ and we can apply our inductive hypothesis, obtaining from $y^{\prime}$ an element $y$ with the desired property. We obtain $z$ similarly.

Corollary 7. If $x$ is not conjugate in $G^{\prime}$ to an element in $A(i, i)$ for any $i$ then $x^{l}$ is not conjugate in $G(l, m)$ to $x^{m}$.

Proof. Let $x$ be in $A(j, k)$ and $y, z$ have the relation to $x$ described in Corollary 6. If $x^{l}$ is conjugate in $G(l, m)$ to $x^{m}$ then there is an integer $q$ and an element $D$ in $G^{\prime}$ such that $a^{-q} x^{l} a^{q}=D^{-1} x^{m} D$. Now there are $u, v$ in $A(j, k)$ such that $x=u^{-1} y u, x=v^{-1} z v$. Hence 


$$
a^{-q} u^{-1} y^{l} u a^{q}=D^{-1} u^{-1} y^{m} u D, \quad a^{-q} v^{-1} z^{l} v a^{q}=D^{-1} v^{-1} z^{m} v D .
$$

Transposing and conjugating by $a^{q}$ yields

$$
\begin{aligned}
& a^{-q} y^{l} a^{q}=\left(a^{-q} u a^{q} D^{-1} u^{-1}\right) y^{m}\left(u D a^{-q} u^{-1} a^{q}\right), \\
& a^{-q} z^{l} a^{q}=\left(a^{-q} v a^{q} D^{-1} v^{-1}\right) z^{m}\left(v D a^{-q} v^{-1} a^{q}\right) .
\end{aligned}
$$

Now for some choice of integers $e, f$ and $g, b, u D a^{-q} u^{-1} a^{q}$ is in $A(e, f)$, $v D a^{-q} v^{-1} a^{q}$ is in $A(g, b)$.

Case 1. $q \geq 0 ; a^{-q} z^{l} a^{q}$ is in $A\left(j-q, k^{\prime}\right)$. Since $z$ is left cyclically reduced in $A\left(j, k^{\prime}\right)$ and $\lambda_{j_{k}},(z) \geq 2$ by Corollary $3 a^{-q} z^{l} a^{q}$ is not conjugate in any left extension of $A\left(j-a, k^{\prime}\right)$ to $z^{m}$. In particular choose $p=\min (g, j-q)$ and also let $r=\max \left(b, k^{\prime}\right)$. If $x^{l}$ is conjugate in $G(l, m)$ to $x^{m}$ then $a^{-q} z^{l} a^{q}$ is conjugate in $A(p, r)$ to $z^{m}$. Since $z^{m}$ and $a^{-q} z^{l} a^{q}$ are left cyclically reduced in $A\left(p, k^{\prime}\right)$ and $\lambda_{p_{k}{ }^{\prime}}\left(z^{m}\right) \geq 2$, if $z^{m}$ is conjugate in $A(p, r)$ to $a^{-q} z^{l} a^{q}$ then by Corollary $5, z^{m}$ is conjugate in $A\left(p, k^{\prime}\right)$ to $a^{-q} z^{l} a^{q}$. However, we have shown that $a^{-q} z^{l} a^{q}$ is not conjugate to $z^{m}$ in $A\left(p, k^{\prime}\right)$ for $p \leq j-q$.

Case 2. $q<0$; apply an analogous argument on $y$.

It is immediate from Corollary 7 that

Corollary 8. If $B$ is in $G^{\prime}$ then $B^{l}$ is conjugate to $B^{m}$ iff $B$ is conjugate to $x^{k}$ for some $i$ and $k$.

3. The endomorphisms of $G(l, m)$.

Proof of Theorem 1 . Note that the defining relators have $a$-exponent sum zero so all relators have $a$-exponent sum zero which puts $B$ in $G^{\prime}$. In fact $G^{\prime}$ and hence $G^{\prime \prime}$ are fully invariant. Now $A^{-1} B^{l} A=B^{m}$, so by Corollary $8, B$ is conjugate in $G^{\prime}$ to $x_{c}^{k}$ and hence in $G(l, m)$ to $b^{k}$. Dividing by $G^{\prime \prime}$ preserves the $a$-exponent on $A$. Every element in $G(l, m) / G^{\prime \prime}$ has the form $r a^{n}$ where $r$ is in $Q(l, m)$ of Lemma 4. Conjugation by $a^{n}$ is seen to act on $Q(l, m)$ as multiplication by $(m / l)^{n}$. Letting $A \equiv r a^{n} \bmod G^{\prime \prime}$ and noting $b^{k} \equiv k \bmod G^{\prime \prime}$ reveals that when $A^{-1} B^{l} A=B^{m}$ is viewed $\bmod G^{\prime \prime}$ the consequence is $a^{-n} r l k r a^{n}=m k$ and hence $(m / l)^{n} k=m k$. Since $B \neq 1, k \neq 0$, so we have $n=1$. Thus $D A D^{-1}=c a$ where $c$ is in $G^{\prime}$. Now $a^{-1} c^{-1} b^{l k} c a=b^{m k}=a^{-1} b^{l k} a$ so $c$ is in the centralizer of $b^{l k}$.

4. Fully invariant kernels. The proofs of Lemmas 6 and 7 below follow from Lemma 3 by a straightforward computation.

Lemma 6. For $n \neq 0, k \geq 0$,

(1) $x_{0}^{n}$ is in $A(k, k)$ iff $m^{k} / n$,

(2) $x_{0}^{n}$ is in $A(-k,-k)$ iff $m^{k} / n$. 
Lemma 7. For each $n \neq 0$ there exist unique integers $p \leq 0 \leq k$ such that $x_{0}^{n}$ is in $A(i$, i) iff $p \leq i \leq k$.

Lemma 8. Let $G=A *_{H} B$. Suppose $a$ is in Center $(A)$ and not in $H$. If $g^{-1} a g$ is in $A$, then $g$ is in $A$.

Proof. If $g$ has representative length zero $g$ is in $A$. Suppose the result for $g$ of length $<n$ and let $g=b g_{1} \cdots g_{n}$. Note that $g_{1}$ is in $A$ for otherwise $g^{-1} a g$ has length $1+2 n$. Now $a$ in Center $(A)$ and the inductive hypothes is yield the result

Lemma 9. Let $G=C *_{K}\left(A *_{H} B\right)=\left(C *_{K} A\right) *_{H} B$. If $a$ is in Center $(A)$ and not in $K, H$ then $g^{-1} a g=a$ implies $g$ in $A$.

Proof. View $G$ with respect to the factors $C$ and $A *_{H} B$. If $g$ has length zero $g$ is in $A$. Suppose $g=k g_{1} \cdots g_{n}$ and assume the result for lengths $<n$. Note $g_{1}$ is in $A *_{H} B$ since $g^{-1} a g$ has length 1 . If $n>1$ then $g_{1}$ is in $K$ for otherwise $g^{-1}$ ag has length $\geq 3$ and so by Lemma 8 and the inductive hypothesis the result holds. If $n=1, g$ is in $A *_{H} B$ and Lemma 8 yields the result.

Lemma 10. Let $H_{i}$ be the subgroup generated by $x_{i}^{l}$ The Center $(A(p, k))$, $p \leq 0 \leq k$, is $\bigcap_{i=p}^{k-1} H p$ if $p \neq k$ and $A(p, p)$ for $p=k$.

Proof. Take induction on $k-p$ applying [4, Corollary 4.5].

Lemma 11. The centralizer of $\left(x_{0}^{l}\right)^{j}, j \neq 0$, in $G^{\prime}$ is $A(p, k)$ where $p, k$ are given in Lemma 7 for $n=l j$.

Proof. View $G^{\prime}$ as

$$
A\left(-\infty, p^{-1}\right) \underset{H_{p-1}^{*}}{*}\left(A(p, k) \underset{H_{k}}{*} A(k+1,+\infty)\right)
$$

and note $x_{0}^{n}$ is not in $H_{k}, H_{p-1}$. Hence if $x_{0}^{n}$ is in Center $(A(p, k))$ then $A(p, k)$ is the centralizer of $x_{0}^{n}$ in $G^{\prime}$, by Lemma 9. Now $x_{0}^{n}$ is in $A(i, i)$ for $p \leq i \leq k$ and so in $H_{i}$ for $p \leq i \leq k-1$. By Lemma $10, x_{0}^{n}$ is in Center $(A(p, k))$.

Lemma 12. Let $\eta$ be as in Lemma 1. If $n=l j, j \neq 0$, and $c$ in $G^{\prime}$ commutes with $b^{n}$ then $c \eta$ commutes with $b^{n}$.

Proof. Let $A(p, k)$ be the centralizer of $b^{n}$ in $G^{\prime}$ as in Lemma 11 and observe that $A(p, k) \eta \subseteq A(p, k)$.

Proof of Theorem 2. We show for any endomorphism $\tau, N \tau \subseteq N$. For $\tau$ : $a \rightarrow a, b \rightarrow 1$, we have $N \tau=1$. Suppose $\tau$ is as in Theorem 1. Note that $N \tau \subseteq N$ iff $D W(a, b)_{\tau} D^{-1}$ and $D V(a, b)_{\tau} D^{-1}$ are in $N$. Now

$$
D W(a, b)_{\tau} D^{-1}=W\left(c a, b^{k}\right), \quad D V(a, b)_{\tau} D^{-1}=V\left(c a, b^{k}\right) .
$$


It follows directly from Lemma 12 that $W\left(c a, b^{k}\right) \eta=1=V\left(c a, b^{k}\right) \eta$.

5. The generalized Hopfian problem. Let $P$ be a property. $G$ is said to be non-Hopfian in the $P$-sense iff there is a proper normal subgroup $N$ possessing property $P$ such that $G / N$ is isomorphic to $G$. Thus the groups in $\mathcal{Q}$ are nonHopfian in the fully invariant sense. It is pointed out in [1] that reduced free groups are Hopfian in the fully invariant sense. In fact it follows from [4, Theorem 3.3] that free groups of arbitrary rank are Hopfian in the characteristic sense.

Problem. Are there groups Hopfian in the fully invariant sense but non-Hopfian in the characteristic sense?

The author wishes to thank Professor Donald Solitar for his suggestions during the writing of [1a] where these results can be found, Professor Wilhelm Magnus for allowing the author to present this work at his Group Theory Seminar, and Professor Peter Stebe for helpful discussions.

\section{REFERENCES}

1. M. Anshel, The endomorphisms of certain one-relator groups and the generalized Hopfian problem, Bull. Amer. Math. Soc. 77 (1971), 348-350. MR 42 \#7757.

1a. Michael Anshel (Orleck), Non-Hopfian groups with fully invariant kemels, Ph.D. Thesis, Adelphi University, Garden City, N.Y., 1967.

2. G. Baumslag and D. Solitar, Some two-generator one-relator non-Hopfian groups, Bull. Amer. Math. Soc. 68 (1962), 199-201. MR 26 \#204.

3. G. Baumslag, Residually finite one-relator groups, Bull. Amer. Math. Soc. 73 (1967), 618-620. MR $35 \# 2953$.

4. W. Magnus, A. Karrass and D. Solitar, Combinatorial group theory: Presentations of groups in terms of generators and relations, Pure and Appl. Math., vol. 13, Interscience, New York, 1966. MR 34 \#7617.

DEPARTMENT OF COMPUTER SCIENCES, CITY COLLEGE (CUNY), NEW YORK, NEW YORK 10031 